\title{
Professores com vínculo temporário apresentam maior frequência de consumo de alimentos pré-preparados
}

\author{
Teachers on temporary employment contracts reveal more frequent \\ consumption of pre-prepared food
}

Douglas Fernando Dias (https://orcid.org/0000-0003-0266-4209) ${ }^{1}$

Francine Nesello Melanda (https://orcid.org/0000-0002-5692-0215) ${ }^{2}$

Elizângela Santana dos Santos (https://orcid.org/0000-0003-4602-1926) ${ }^{3}$

Selma Maffei de Andrade (https://orcid.org/0000-0001-6843-8330) ${ }^{4}$

Arthur Eumann Mesas (https://orcid.org/0000-0002-0088-8607) ${ }^{4}$

Alberto Durán González (https://orcid.org/0000-0002-4203-9400) ${ }^{4}$
${ }^{1}$ Programa de PósGraduação em Enfermagem, Universidade Estadual de

Londrina (UEL). Av. Robert Koch 60, Vila Operária. 86039-440 Londrina PR Brasil.betoduran@uel.br ${ }^{2}$ Instituto de Saúde Coletiva, Universidade Federal do

Mato Grosso. Cuiabá MT

Brasil.

${ }^{3}$ Universidade Estadual

do Norte do Paraná.

Jacarezinho PR Brasil.

${ }^{4}$ Programa de Pós-

Graduação em Saúde

Coletiva, UEL. Londrina PR

Brasil.

\begin{abstract}
The scope of this article is to analyze the eating behavior of schoolteachers according to the type of employment contract. Interviews were conducted at 20 public state schools in Londrina, State of Paraná, between 2012 and 2013. Of the 978 teachers interviewed, 672 (68.7\%) had permanent employment contracts and 306 (31.3\%) were on temporary employment contracts. High frequencies of recommended eating behaviors consumption of fruit, vegetables and/or vegetables and removing visible fat from red meat - were observed in teachers with a permanent employment contract. On the other hand, teachers with temporary employment contracts revealed a high frequency of non-recommended eating behaviors with consumption of pre-prepared foods and packaged drinks or sodas. It was highlighted that a high frequency of consumption of pre-prepared foods was statistically associated with the type of employment contract irrespective of sociodemographic, lifestyle and health status factors.
\end{abstract}

Key words Eating behavior, Schoolteachers, Occupational health, Employment conditions
Resumo $O$ artigo tem por objetivo analisar as condutas alimentares de professores segundo os vínculos de trabalho. As entrevistas foram realizadas em 20 escolas da rede estadual de Londrina entre 2012 e 2013. Dos 978 professores entrevistados, 672 (68,7\%) tinham vinculo efetivo e 306 (31,3\%) temporário. Altas frequências de condutas alimentares recomendadas - consumo de frutas, verduras e/ou legumes e retirada da gordura visivel da carne vermelha - foram observadas em professores com vinculo efetivo. Em contrapartida, professores com vínculo temporário apresentaram altas frequências de condutas alimentares não recomendadas-consumo de alimentos pré-preparados e de bebidas industrializadas ou refrigerantes. Destaca-se que o consumo em alta frequência de alimentos pré-preparados esteve estatisticamente associado ao tipo de vinculo independentemente de fatores sociodemográficos, de estilo de vida e da condição de saúde.

Palavras-chave Comportamento alimentar, Docentes, Saúde do trabalhador, Condições de trabalho 


\section{Introdução}

A alimentação é objeto de estudo de diferentes áreas do conhecimento e se configura como um ato social que tem interferência de costumes, protocolos e situações. Os padrões tanto de permanência quanto de mudança de hábitos alimentares são frutos da dinâmica social, influenciados pelas praticidades oferecidas pelas tecnologias e o modo de vida, que se refletem nas escolhas alimentares ${ }^{1}$.

A partir da segunda metade do século XX, o Brasil passou por significativas mudanças econômicas, culturais e sociais e, em decorrência, observou-se mudanças no mercado de trabalho, estilos de vida e demandas nutricionais ${ }^{2}$. Tendo em vista essas mudanças, Diez Garcia ${ }^{3}$ aponta que é imposto ao indivíduo a necessidade de ajuste de atividades cotidianas segundo as condições das quais dispõe, como tempo, recursos financeiros, locais disponíveis para se alimentar, local e periodicidade das compras, entre outras. Com isso, as soluções são capitalizadas pela indústria e comércio, delineando novas modalidades no modo de comer, caracterizada pela falta de tempo para preparo e consumo de alimentos, produtos gerados com novas técnicas de conservação e preparo e pelo deslocamento das refeições de casa para restaurantes e lanchonetes ${ }^{3}$.

Sabe-se que a alimentação rica em frutas, verduras e legumes e pobre em gorduras e sódio constitui-se fator protetor para diversas doenças como síndrome metabólica, hipertensão arterial e diabetes ${ }^{4-7}$. No entanto, diante do novo cenário, alimentos industrialmente processados são cada vez mais consumidos devido à facilidade de preparo. Dentro dessa classificação, incluemse os alimentos ultraprocessados, que são práticos para consumo e feitos para serem duráveis e atrativos ${ }^{8}$. Todavia, esses produtos com frequência apresentam alto teor de gorduras, açúcares e sódio e também tendem a ser pobres em fibras ${ }^{7}$.

A classe trabalhadora, em geral, é a mais afetada pelas constantes mudanças na sociedade. Nesse contexto, destaca-se o trabalho docente, que se caracteriza por ser desgastante, marcado por uma expressiva carga horária em sala de aula, excesso de tarefas burocráticas, falta de autonomia e de infraestrutura adequada no ambiente escolar, tornando evidente o quadro crônico de depreciação e desqualificação social dos professores? ${ }^{9}$ Essa situação é ainda agravada com a precarização do vínculo de trabalho na carreira docente, como o contrato por tempo determinado. O vínculo por meio de contratos temporários precários, que não conduzem à estabilidade e à progressão profissional, tem efeitos importantes na profissionalização docente, gerando rodízio excessivo de professores, instabilidade das equipes, absenteísmos e desistências da profissão ${ }^{10}$. Para além das consequências ocupacionais, uma metanálise apontou que trabalhadores com contratos menos estáveis apresentam piores indicadores de saúde quando comparados àqueles com contratos estáveis ${ }^{11}$.

Acredita-se que a sobrecarga do trabalho pode influenciar na alimentação do docente, com prejuízo possivelmente maior aos professores temporários. Compreender essa relação pode contribuir na identificação de um grupo mais vulnerável a más condutas alimentares e, com isso, planejar políticas adequadas de prevenção de determinadas doenças ligadas a essa situação no futuro e promover a melhoria das condições de trabalho de professores, como o tipo de vínculo. Assim, o objetivo deste estudo foi analisar as condutas alimentares relatadas pelos professores da rede estadual de Londrina segundo o tipo de vínculo de trabalho (efetivo e temporário) e verificar se as associações se mantêm após análises ajustadas por variáveis sociodemográficas, de estilo de vida e de condições de saúde.

\section{Métodos}

Estudo epidemiológico observacional do tipo transversal vinculado ao projeto "Saúde, estilo de vida e trabalho de professores da rede pública do Paraná - PRÓ MESTRE”, coordenado por docentes do programa de pós-graduação em Saúde Coletiva da Universidade Estadual de Londrina (UEL).

Todos os professores das 20 escolas com maior número de docentes do município de Londrina foram convidados a participar o estudo. Essas escolas foram selecionadas por conveniência, por estarem localizadas na área urbana e serem de mais fácil acesso. Essa população representa aproximadamente $70 \%$ do número total de professores atuantes no ensino regular do município.

Neste estudo, foram incluídos os professores que exerciam atividade docente no ensino fundamental ou médio. Os professores excluídos da pesquisa foram aqueles que estavam em readaptação ou afastados da função durante o período de coleta. Foram classificados como perdas os professores afastados por licença médica que não retornaram para a atividade docente após 30 dias 
da conclusão das entrevistas em seu colégio, que não foram localizados para o agendamento da entrevista após cinco tentativas e os que se recusaram a participar.

Realizou-se estudo piloto em cidade vizinha a fim de testar os instrumentos e a logística de coleta de dados. Os entrevistadores foram previamente treinados e orientados quanto à condução das entrevistas. A coleta de dados ocorreu de agosto a dezembro de 2012 e fevereiro a junho de 2013, mediante a aplicação de instrumentos constituídos por um formulário (para anotação de dados) e um questionário preenchido pelo próprio professor após entrevista. Durante as entrevistas foram obtidos dados sobre condutas alimentares, estilo de vida e condições de saúde. Dados sociodemográficos foram preenchidos pelo professor no questionário. As entrevistas tiveram duração aproximada de 40 minutos e o preenchimento do questionário cerca de $10 \mathrm{mi}$ nutos.

A pesquisa foi realizada mediante assinatura do termo de consentimento livre e esclarecido (TCLE), obedecendo todos os critérios éticos de pesquisa envolvendo seres humanos. O projeto de pesquisa foi aprovado pelo comitê de ética em pesquisa da UEL.

Devido à falta de instrumentos padronizados, informações sobre condutas alimentares foram elaboradas pelos pesquisadores com base em outros estudos sobre o tema ${ }^{12-18}$. Definidas como variáveis dependentes, as condutas alimentares foram divididas em recomendadas e não recomendadas. Optou-se por utilizar essa denominação porque as evidências na literatura para afirmar se determinadas condutas são prejudiciais ou protetoras da saúde ainda são insuficientes, mas, ainda assim, são utilizadas nos guias alimentares para orientar a adoção de uma alimentação mais saudável. As condutas recomendadas utilizadas neste estudo foram: a) consumo de frutas, b) consumo de verduras e/ou legumes, c) retirada da gordura visível quando come carne vermelha e d) retirada da pele da carne de frango. As condutas alimentares não recomendadas abordadas neste estudo foram: a) substituição de ao menos uma das refeições por lanches, b) consumo de alimentos pré-preparados, c) consumo de bebidas industrializadas, d) comer assistindo televisão ou em frente ao computador e e) comer salgadinhos ou doces entre as refeições principais. Os professores responderam a frequência com que adotavam cada uma das condutas alimentares e tal informação foi usada para criar as seguintes categorias de análise: a) baixa ou média frequência (nunca a duas vezes na semana) e b) alta frequência (três vezes na semana a diariamente).

O tipo de vínculo, autorreferido como efetivo ou temporário, compôs a variável independente principal. As variáveis sociodemográficas usadas como ajuste foram: sexo (masculino; feminino), idade (contínua) e renda familiar mensal (até R\$ 5.000,00 e R \$ 5.001,00 ou mais). As variáveis de estilo de vida e condições de saúde foram: inatividade física no tempo livre, ou seja, nenhuma atividade física no tempo livre ( $\mathrm{sim}$; não), Índice de Massa Corporal (IMC) em $\mathrm{Kg} / \mathrm{m}^{2}$ calculado após relato de peso e altura, categorizado em (baixo peso ou normal - $\leq 24,9$; sobrepeso ou obesidade $\geq 25,0$ ), relato de diagnóstico médico de hipertensão arterial, diabetes melito e hiperlipidemia ( $\operatorname{sim}$; não).

Os dados foram duplamente digitados e as inconsistências foram corrigidas. Para a análise descritiva das variáveis categóricas utilizaram-se medidas de frequências absoluta e relativa, e para a análise bivariada foi utilizado o teste do Quiquadrado. Idade, única variável contínua, foi descrita por meio da mediana e da distância interquartil. Na análise bivariada, foi realizado teste de Kolmogorov-Smirnov e, verificada a não normalidade, foi realizado o teste de Mann-Whitney para verificação de diferença estaticamente significativa entre as medianas.

As condutas alimentares investigadas foram consideradas individualmente como variáveis dependentes. Assim, na análise de múltiplos fatores, foi construído um modelo de ajuste entre tipo de vínculo de trabalho e cada uma das condutas alimentares, com inserção das variáveis sociodemográficas, relacionados ao estilo de vida e as de condições de saúde. As razões de prevalência bruta e as derivadas de análises de múltiplos fatores foram obtidas por meio da regressão de Poisson com ajuste robusto de variância, com utilização da razão de prevalência (RP) como medida de associação, e adotado nível de significância de 5\% com apresentação do p-valor e do intervalo de confiança (IC) de 95\%. As análises foram realizadas no programa SPSS versão 19.0.

\section{Resultados}

Dentre os 1.505 professores que trabalhavam nas 20 escolas participantes da pesquisa, 379 foram excluídos por atuarem apenas no ensino técnico ou complementar ou por estarem afastados ou readaptados. Dos 1.126 professores elegíveis, 65 
(5,8\%) estavam de licença, 63 (5,6\%) se recusaram a participar da pesquisa e $20(1,8 \%)$ não foram encontrados após a quinta tentativa de contato. Portanto, foram entrevistados 978 professores (taxa de resposta $=86,9 \%$ ).

Os professores com vínculo temporário representavam $31,1 \%(\mathrm{n}=304)$ do total. A idade apresentou mediana de 42,0 anos (distância interquartil de 16,0). A maioria dos professores era do sexo feminino $(68,5 \%)$ e tinham renda familiar mensal de até R\$ 5.000,00. Pouco mais da metade da população estudada relatou não praticar atividade física no lazer e apresentava sobrepeso ou obesidade. A prevalência de relatos de diagnóstico médico para hipertensão e hiperlipidemia foram de aproximadamente $15,0 \%$ e a de diabetes melito, 4,4\% (Tabela 1).

As condutas recomendadas apresentaram prevalências que variaram de $44,9 \%$ (consumo de frutas) a 60,0\% (retirada da pele da carne de frango). Professores com vínculo efetivo apresentaram maior prevalência de todas as condutas recomendadas em alta frequência em relação aos temporários, exceto para a variável retirada da pele da carne de frango (Tabela 2).

Em relação às condutas alimentares não recomendadas, as frequências relativas variaram de $10,4 \%$ (consome alimentos pré-preparados) a $35,4 \%$ (consome bebidas industrializadas ou refrigerantes). As prevalências de consumo de alimentos pré-preparados, consumo de bebidas industrializadas/refrigerantes e comer assistindo televisão ou em frente ao computador foram superiores entre professores temporários em relação aos efetivos. Enquanto que para as condutas substituir refeições por lanches e consomir salgadinhos ou doces entre as refeições principais, não foram observadas diferenças estatisticamente significativas entre os tipos de vínculos (Tabela 3 ).

$\mathrm{Na}$ análise bruta, o consumo em alta frequência das condutas recomendadas foi inferior entre os professores temporários quando comparados aos efetivos - consumo de frutas $(\mathrm{RP}=0,82$; IC95\%0,70-0,97), consumo de verduras e/ou legumes $(\mathrm{RP}=0,80$; IC95\%0,70-0,91) e retirada da gordura visível quando come carne vermelha $(\mathrm{RP}=0,78$; IC95\%0,67-0,91). A retirada da pele da carne de frango não se mostrou associada ao tipo de vínculo. No que diz respeito às condutas não recomendadas, o consumo em alta frequência teve proporção superior nos temporários para as seguintes variáveis: consumo de alimentos pré-preparados ( $\mathrm{RP}=1,90$; IC95\%1,32-2,73), consumo de bebidas industrializadas/refrigerantes $(\mathrm{RP}=1,22$; IC95\%1,03-1,46) e comer assis- tindo TV/computador ( $\mathrm{RP}=1,25$; IC95\%1,041,51). Na análise ajustada, apenas consumo de alimentos pré-preparados manteve-se associado ao vínculo temporário independentemente de fatores sociodemográficos, de estilo de vida e da condição de saúde (RP = 1,58; IC95\%1,04-2,34) (Tabela 4).

\section{Discussão}

De todas as condutas alimentares investigadas, apenas consumo de alimentos pré-preparados manteve-se associado ao vínculo temporário após ajustes por variáveis de confundimento. No entanto, embora as demais associações estatisticamente significativas nas análises bivariadas não tenham se mantido após ajustes, destaca-se que, de modo geral, professores com vínculo temporário apresentaram piores condutas alimentares, haja vista que em tal grupo observou-se frequências inferiores das condutas recomendadas e superiores das não recomendadas.

Tanto a proporção de mulheres $(68,5 \%)$ quanto a média de idade (41 anos) encontradas neste estudo foram semelhantes aos valores observados em amplo estudo conduzido com professores de 34 países, $68 \%$ e 43 anos, respectivamente $^{19}$. Contudo, o percentual de professores com vínculo efetivo encontrado em nosso estudo $(68,9 \%)$ foi menor que a média mundial $(82,5 \%)$ e brasileira $(76,5 \%)$ no ano de $2013^{12}$. Para Nova$\mathrm{es}^{20}$, professores temporários apresentam vínculos contratuais extremamente frágeis e não gozam da mesma estabilidade no emprego que os efetivos, vivendo, ano a ano, a insegurança e a instabilidade relacionadas à possibilidade de se manterem empregados, com impactos sobre a maneira de conceber a escola, a profissão e o ensino. Portanto, esse resultado sugere uma maior precarização nas relações trabalhistas na população estudada, o que fortalece a importância do estudo.

O consumo de alimentos pré-preparados foi maior entre os temporários. Tendo em vista que o consumo deste tipo de alimento é crescente em populações de menor poder aquisitivo ${ }^{21}$ e que professores temporários, em geral, apresentam menor renda mensal (Tabela 1 ), a primeira hipótese foi a de que a variável renda explicaria tal associação também na população desta pesquisa. A associação entre a variável renda familiar e as condutas alimentares foi verificada em diversos estudos $^{14,22,23}$. Todavia, quando tal associação foi ajustada pela renda, não houve modificação significativa no valor da RP. 
Tabela 1. Características gerais da amostra segundo tipo de vínculo de trabalho de professores da rede estadual de Londrina-PR, 2012-2013 ( $\mathrm{n}=978$ ).

\begin{tabular}{|c|c|c|c|c|c|c|c|}
\hline & \multicolumn{2}{|c|}{ Total } & \multicolumn{2}{|c|}{ Efetivo } & \multicolumn{2}{|c|}{ Temporário } & Valor de $p$ \\
\hline \multicolumn{8}{|l|}{ Idade (em anos) } \\
\hline \multirow[t]{3}{*}{ Mediana (distância interqualtil) } & \multicolumn{2}{|c|}{$42,0(16,0)$} & \multicolumn{2}{|c|}{$44,0(13,0)$} & \multicolumn{2}{|c|}{$33,0(14,0)$} & $<0,001$ \\
\hline & \multicolumn{2}{|c|}{ Total } & \multicolumn{2}{|c|}{ Efetivo } & \multicolumn{2}{|c|}{ Temporário } & \multirow{2}{*}{ Valor de p } \\
\hline & $\mathbf{n}$ & $\%$ & $\mathbf{n}$ & $\%$ & $\mathbf{n}$ & $\%$ & \\
\hline Sexo & & & & & & & 0,628 \\
\hline Masculino & 308 & 31,5 & 209 & 31,0 & 99 & 32,6 & \\
\hline Feminino & 670 & 68,5 & 465 & 69,0 & 205 & 67,4 & \\
\hline Renda mensal familiar ${ }^{\mathrm{A}, \mathrm{B}}$ & & & & & & & $<0,001$ \\
\hline Até $\mathrm{R} \$ 5.000,00$ & 576 & 59,4 & 342 & 59,4 & 234 & 40,6 & \\
\hline $\mathrm{R} \$ 5.001,00$ ou mais & 393 & 40,6 & 326 & 83,0 & 67 & 17,0 & \\
\hline Atividade física no tempo livre & & & & & & & 0,002 \\
\hline $\operatorname{Sim}$ & 459 & 46,9 & 339 & 50,3 & 120 & 39,5 & \\
\hline Não & 519 & 53,1 & 335 & 49,7 & 184 & 60,5 & \\
\hline Índice de Massa Corporal ${ }^{\mathrm{A}}$ & & & & & & & 0,035 \\
\hline Baixo peso ou normal & 468 & 48,1 & 308 & 45,8 & 160 & 53,2 & \\
\hline Sobrepeso e obesidade & 505 & 51,9 & 364 & 54,2 & 141 & 46,8 & \\
\hline Hipertensão & & & & & & & $<0,001$ \\
\hline Sim & 147 & 15,0 & 122 & 18,1 & 25 & 8,2 & \\
\hline Não & 831 & 85,0 & 552 & 81,9 & 279 & 91,8 & \\
\hline Diabetes melito & & & & & & & 0,032 \\
\hline $\operatorname{Sim}$ & 43 & 4,4 & 36 & 5,3 & 7 & 2,3 & \\
\hline Não & 935 & 95,6 & 638 & 94,7 & 297 & 97,7 & \\
\hline Hiperlipidemia & & & & & & & 0,002 \\
\hline Sim & 151 & 15,4 & 120 & 17,8 & 31 & 10,2 & \\
\hline Não & 827 & 84,6 & 554 & 82,2 & 273 & 89,8 & \\
\hline
\end{tabular}

Tabela 2. Prevalência de alta frequência de condutas alimentares recomendadas segundo tipos de vínculo de trabalho de professores da rede estadual de Londrina-PR, 2012-2013 $(\mathrm{n}=978)$.

\begin{tabular}{|c|c|c|c|c|c|c|c|}
\hline \multirow{2}{*}{ Condutas recomendadas } & \multicolumn{2}{|c|}{ Total } & \multicolumn{2}{|c|}{ Efetivo } & \multicolumn{2}{|c|}{ Temporário } & \multirow{2}{*}{$\begin{array}{l}\text { Valor } \\
\text { de p }\end{array}$} \\
\hline & $\mathrm{n}$ & $\%$ & $\mathbf{n}$ & $\%$ & n & $\%$ & \\
\hline Consome frutas & 439 & 44,9 & 320 & 47,5 & 119 & 39,1 & 0,015 \\
\hline Consome verduras e/ou legumes & 558 & 57,1 & 410 & 60,8 & 148 & 48,7 & $<0,001$ \\
\hline Retira a gordura visível quando come carne vermelha* & 468 & 50,0 & 349 & 53,5 & 119 & 41,9 & 0,001 \\
\hline Retira a pele da carne de frango ${ }^{\star *}$ & 556 & 60,0 & 397 & 61,9 & 159 & 55,8 & 0,078 \\
\hline
\end{tabular}

${ }^{\star} \mathrm{n}=936{ }^{\star *} \mathrm{n}=926$.

Uma segunda hipótese para esse achado seria de que professores temporários são mais jovens, e que essa característica, tem forte relação com a conduta alimentar, como verificado em diversos estudos $^{14-15,22,24}$. Com o avançar da idade, pode haver maior preocupação com a saúde e, portanto, com a prevenção de doenças crônicas como hipertensão arterial, diabetes mellitus, dislipidemias e outras que estão diretamente relacionadas com a alimentação. Além disso, diferentemente dos mais jovens, a população mais velha viveu em um momento em que a cultura alimentar do país não tinha o consumo de alimentos processados e com alto teor de gordura tão marcante ${ }^{25}$. Essa 
Tabela 3. Prevalência de alta frequência de condutas alimentares não recomendadas segundo tipos de vínculo de trabalho de professores da rede estadual de Londrina-PR, 2012-2013 ( $\mathrm{n}=978$ ).

\begin{tabular}{|c|c|c|c|c|c|c|c|}
\hline \multirow{2}{*}{ Condutas não recomendadas } & \multicolumn{2}{|c|}{ Total } & \multicolumn{2}{|c|}{ Efetivo } & \multicolumn{2}{|c|}{ Temporário } & \multirow{2}{*}{$\begin{array}{l}\text { Valor } \\
\text { de p }\end{array}$} \\
\hline & $\mathbf{n}$ & $\%$ & $\mathbf{n}$ & $\%$ & $\mathbf{n}$ & $\%$ & \\
\hline Substitui refeições por lanches * & 209 & 21,4 & 142 & 21,1 & 67 & 22,1 & 0,713 \\
\hline Consome alimentos pré-preparados & 102 & 10,4 & 55 & 8,2 & 47 & 15,4 & 0,001 \\
\hline Consome bebidas industrializadas ou refrigerantes & 346 & 35,4 & 223 & 33,1 & 123 & 40,5 & 0,026 \\
\hline Come salgadinhos ou doces entre refeições principais & 145 & 14,8 & 99 & 14,7 & 46 & 15,1 & 0,857 \\
\hline Come assistindo TV ou usando o computador & 310 & 31,7 & 198 & 29,4 & 112 & 36,8 & 0,020 \\
\hline
\end{tabular}
${ }^{*} \mathrm{n}=977$.

Tabela 4. Razões de prevalência bruta e ajustada das condutas alimentares adotadas com alta frequência segundo o tipo de vínculo de professores da rede estadual de Londrina-PR, 2012-2013.

\begin{tabular}{|c|c|c|c|c|}
\hline \multirow[b]{2}{*}{ Variáveis dependentes } & \multicolumn{2}{|c|}{ Análise bruta } & \multicolumn{2}{|c|}{ Análise ajustada } \\
\hline & $\begin{array}{c}\text { RP } \\
(\text { IC 95\%) }\end{array}$ & $\begin{array}{l}\text { Valor } \\
\text { de p }\end{array}$ & $\begin{array}{c}\text { RP } \\
(\text { IC 95\%) }\end{array}$ & $\begin{array}{l}\text { Valor } \\
\text { de p }\end{array}$ \\
\hline \multicolumn{5}{|l|}{ Condutas recomendadas } \\
\hline Consumo de frutas & $\begin{array}{r}0,82 \\
(0,70-0,97)\end{array}$ & 0,019 & $\begin{array}{r}1,08 \\
(0,91-1,28)\end{array}$ & 0,376 \\
\hline Consumo de verduras e/ou legumes & $\begin{array}{r}0,80 \\
(0,70-0,91)\end{array}$ & 0,001 & $\begin{array}{r}0,97 \\
(0,85-1,11)\end{array}$ & 0,668 \\
\hline Retira a gordura visível quando come carne vermelha & $\begin{array}{r}0,78 \\
(0,67-0,91)\end{array}$ & 0,002 & $\begin{array}{r}0,86 \\
(0,73-1,02)\end{array}$ & 0,074 \\
\hline Retira a pele da carne de frango & $\begin{array}{r}0,90 \\
(0,80-1,02)\end{array}$ & 0,087 & $\begin{array}{r}0,99 \\
(0,87-1,13)\end{array}$ & 0,897 \\
\hline \multicolumn{5}{|l|}{ Condutas não recomendadas } \\
\hline Substitui refeições por lanches & $\begin{array}{r}1,50 \\
(0,81-1,36)\end{array}$ & 0,712 & $\begin{array}{r}1,10 \\
(0,82-1,47)\end{array}$ & 0,530 \\
\hline Consumo de alimentos pré-preparados & $\begin{array}{r}1,90 \\
(1,32-2,73)\end{array}$ & 0,001 & $\begin{array}{r}1,58 \\
(1,04-2,34)\end{array}$ & 0,034 \\
\hline Consumo de bebidas industrializadas ou refrigerantes & $\begin{array}{r}1,22 \\
(1,03-1,46)\end{array}$ & 0,023 & $\begin{array}{r}0,96 \\
(0,79-1,16)\end{array}$ & 0,653 \\
\hline Come salgadinhos ou doces entre refeições principais & $\begin{array}{r}1,03 \\
(0,75-1,42)\end{array}$ & 0,857 & $\begin{array}{r}0,78 \\
(0,55-1,11)\end{array}$ & 0,171 \\
\hline Come assistindo TV ou em frente ao computador & $\begin{array}{r}1,25 \\
(1,04-1,51)\end{array}$ & 0,018 & $\begin{array}{r}0,95 \\
(0,77-1,17)\end{array}$ & 0,651 \\
\hline
\end{tabular}

^Tipo de vínculo efetivo foi considerado categoria de referência. Análise ajustada por: idade, sexo, renda, IMC, atividade física no tempo livre, diagnóstico médico de hipertensão arterial, diagnóstico médico de diabetes e diagnóstico médico de hiperlipidemia.

hipótese também foi refutada com a inclusão da variável idade como ajuste, fortalecendo uma terceira explicação, a de que o consumo de alimentos pré-preparados pode ser explicado pelo tipo de vínculo de trabalho em professores.

Considerando que professores com vínculo efetivo têm maior poder de decisão na escolha dos locais que irão trabalhar ${ }^{26}$, e embora não se tenha informações sobre deslocamentos ou localização das escolas em que cada professor trabalha, é possível que as instituições em áreas mais afastadas sejam as que mais frequentemente apresentam-se como possibilidade de locais de trabalho aos temporários, dificultando o acesso ao alimento preparado em casa (pela distância) ou em restaurantes (pela menor densidade). Acrescenta-se a esse cenário, elevada carga horária de trabalho e atuação em maior número de escolas ${ }^{26}$. Nessa perspectiva, o conjunto dessas características pode contribuir com a diminuição 
do tempo disponível para o preparo do alimento e favorecer a escolha daqueles prontos para o consumo.

As demais condutas alimentares, recomendadas e não recomendadas, apesar de associadas ao tipo de vínculo nas análises bivariadas, perderam significância estatística após ajustes. É plausível que, na população do presente estudo, as variáveis incluídas no modelo exerçam forte influência sobre esta relação, especialmente idade e renda, que conhecidamente interferem nas condutas alimentares de diversas populações estudadas ${ }^{14,22-24}$. Entretanto, embora analisadas separadamente, as condutas alimentares constituem um constructo único e representam um mesmo comportamento. Assim, nossos resultados apontam que o vínculo de trabalho, junto à outras características sociodemográficas, de estilo de vida e de condições de saúde, são importantes na determinação de quais condutas alimentares serão adotadas.

Algumas características metodológicas do presente estudo devem ser destacadas. A estratégia de coleta de dados adotada implicou em baixas porcentagens de recusas e de professores que não puderam ser localizados. Além disso, cabe frisar a escassez de pesquisas que relacionem os vínculos de trabalho com as condutas alimentares em professores, o que ressalta a importância desta pesquisa e sua originalidade. $\mathrm{O}$ desenho transversal configura uma limitação do estudo, já que não permite a inferência causal entre as variáveis. No entanto, a direção da associação investigada no presente estudo é plausível do ponto de vista teórico. Há também uma limitação em extrapolar os resultados encontrados para outras populações de professores, especialmente aqueles de escolas de menor porte e localizadas em área rural, uma vez que, em nosso estudo, foram selecionadas aquelas de maior porte da área urbana. Todavia, ressalta-se que a população estudada representava aproximadamente $70 \%$ dos professores do ensino fundamental e médio do município. Deve-se considerar ainda que professores que já adoeceram e com as piores condições de trabalho podem não ter sido entrevistados porque já se afastaram ou saíram do emprego (viés do trabalhador saudável). Assim, podemos afirmar que nossos resultados são conservadores, já que a inclusão dessa população nas análises poderia potencializar as hipóteses investigadas.

Por fim, observou-se que professores com vínculo temporário apresentam piores condutas alimentares, em destaque o consumo de ali- mentos pré-preparados, o qual se manteve associado a esse contrato de trabalho, independente de características sociodemográficas, estilo de vida e condições de saúde. Considerando que no Brasil a proporção de professores com vínculo temporário está acima da média mundial e que este vínculo está associado a piores indicadores de condições, perfil e ambiente de trabalho ${ }^{10}$ e de saúde $^{19}$, salientamos a necessidade da continuidade de pesquisas que abordem o perfil alimentar dos docentes em geral e sua associação com os diferentes tipos de vínculos. Acredita-se que tais estudos possam subsidiar estratégias de enfrentamento desse agravo, tanto pela execução de amplas politicas públicas, que considerem a complexidade e a multifatoriedade do fenômeno, bem como ações específicas para docentes da educação básica, como o favorecimento de vínculos de trabalhos mais seguros e estáveis e a estruturação de ambientes favoráveis a uma alimentação mais saudável.

\section{Colaboradores}

Declaração de autoria: ES Santos, SM Andrade, AE Mesas e AD González participaram do delineamento do estudo. ES Santos e AD González participaram da redação da primeira versão do artigo. DF Dias e FN Melanda apoiaram a depuração do banco de dados e elaboração das análises estatísticas. Todos os autores revisaram e aprovaram a versão final do artigo.

\section{Agradecimentos}

À Fundação Araucária pelo apoio financeiro, à CAPES pela concessão de bolsas de estudo - doutorado e ao CNPq, pela concessão de bolsas de iniciação científica e produtividade em pesquisa.

Às fontes de financiamento: ES Santos recebeu bolsa de iniciação científica do Conselho Nacional de Desenvolvimento Científico e Tecnológico $(\mathrm{CNPq})$. DF Dias e FN Melanda receberam bolsa de estudos - doutorado da Coordenação de Aperfeiçoamento de Pessoal de Nível Superior (CAPES). SM Andrade e AE Mesas receberam bolsa de produtividade em pesquisa do CNPq. Este estudo foi parcialmente financiado pela Fundação Araucária do Paraná. 


\section{Referências}

1. Lima RS, Neto JAF, Farias RCP. Alimentação, comida e cultura: o exercício da comensalidade. Demetra 2015; 10(3):507-522.

2. Batista M, Rissin A. A transição nutricional no Brasil: tendências regionais e temporais. Cad Saude Publica 2003; 19(Supl. 1):181-191.

3. Diez Garcia RW. Reflexos da globalização na cultura alimentar: considerações sobre as mudanças na alimentação urbana. Rev Nutr 2003; 16(4):483-492.

4. Oliveira JEP, Vencio S. Diretrizes da Sociedade Brasileira de Diabetes: 2013-2014/Sociedade Brasileira de Diabetes. São Paulo: AC Farmacêutica; 2014.

5. V Diretrizes Brasileiras de Hipertensão Arterial. Arq Bras Cardiol 2007; 89(3):e24-e79.

6. Brandão AP, Brandão AA, Nogueira AR, Suplicy H, Guimarães JI, Oliveira JEP. I Diretriz Brasileira de Diagnóstico e Tratamento da Síndrome Metabólica. Arq Bras Cardiol 2005; 84(Supl. 1):3-28.

7. Brasil. Ministério da Saúde (MS). Guia alimentar para a população brasileira. Brasília: MS; 2014.

8. Monteiro CA, Levy RB, Claro RM, Castro IRR, Cannon G. A new classification of foods based on the extent and purpose of their processing. Cad Saude Publica 2010; 26(11):2039-2049.

9. Rocha VM, Fernandes MH. Qualidade de vida de professores do ensino fundamental: uma perspectiva para a promoção da saúde do trabalhador. J Bras Psiquiatr 2008; 57(1):23-27.

10. Gatti BA, Barreto ESS, André MEDA. Políticas docentes no Brasil: um estado da arte. Brasília: Unesco; 2011.

11. Sanwald A, Theurl E. Atypical employment and health: A meta-analysis. Viena: Working Papers in Economics and Statistics; 2014.

12. Bes-Rastrollo M, Sanchez-Villegas A, Basterra-Gortari FJ, Nunez-Cordoba JM, Toledo E, Serrano-Martinez M. Prospective study of self-reported usual snacking and weight gain in a Mediterranean cohort: the SUN project. Clin Nutr 2010; 29(3):323-330.

13. Bleich SN, Wang YC, Wang Y, Gortmaker SL. Increasing consumption of sugar-sweetened beverages among US adults: 1988-1994 to 1999-2004. Am J Clin Nutr 2008; 89(1):372-381.

14. Gimeno SGA, Mondini L, Moraes SAD, Freitas ICMD. Padrões de consumo de alimentos e fatores associados em adultos de Ribeirão Preto, São Paulo, Brasil: Projeto OBEDIARP. Cad Saude Publica 2011; 27(3):533545.

15. Pinho CPS, Silva Diniz A, Arruda IKG, Lira PIC, Cabral PC, Siqueira LAS, Batista Filho M. Consumo de alimentos protetores e preditores do risco cardiovascular em adultos do estado de Pernambuco. Rev. Nutr. 2012; 25(3):341-351.
16. Odegaard AO, Koh WP, Arakawa K, Yu MC, Pereira MA. Soft drink and juice consumption and risk of physician-diagnosed incident type 2 diabetes: the Singapore Chinese Health Study. Am J Epidemiol 2010; 171(6):701-708.

17. Rehm CD, Matte TD, Van Wye G, Young C, Frieden TR. Demographic and behavioral factors associated with daily sugar-sweetened soda consumption in New York City adults. J Urban Health 2008; 85(3):375-385.

18. Rombaldi AJ, Neutzling MB, Silva MCD, Azevedo MR, Hallal PC. Fatores associados ao consumo regular de refrigerante não dietético em adultos de Pelotas, RS. Rev Saude Publica 2011; 45(2):382-390.

19. Organization for Economic Cooperation and Development (OECD). TALIS 2013 results: an international perspective on teaching and learning. Paris: OECD; 2014.

20. Novaes LC. A formação des (continuada) dos professores temporários: provisoriedade e qualidade de ensino. Rev Diálogo Educ 2010 10(30):247-265.

21. Martins APB, Levy RB, Claro RM, Moubarac JC, Monteiro CA. Increased contribution of ultra-processed food products in the Brazilian diet (1987-2009). Rev Saude Publica 2013; 47(4):656-665.

22. Mondini L, Moraes SAD, Freitas ICM D, Gimeno SGA. Consumo de frutas e hortaliças por adultos em Ribeirão Preto, SP. Rev Saude Publica 2010; 44(4):686694.

23. Neutzling MB, Rombaldi AJ, Azevedo MR, Hallal PC. Fatores associados ao consumo de frutas, legumes e verduras em adultos de uma cidade no Sul do Brasil. Cad Saude Publica 2009; 25(11):2365-2374.

24. Campos VC, Bastos JL, Gauche H, Boing AF, Assis MAAD. Fatores associados ao consumo adequado de frutas, legumes e verduras em adultos de Florianópolis. Rev Bras Epidemiol 2010; 13(2):352-362.

25. Jaime PC, Figueiredo ICR, Moura ECD, Malta DC. Fatores associados ao consumo de frutas e hortaliças no Brasil, 2006. Rev Saude Publica 2009; 43(Supl. 2):57-64.

26. Ferreira DCK, Abreu CBM. Professores temporários: flexibilização das contratações e condições de trabalho docente. Trabalho \& Educação 2014; 23(2):129139.

Artigo apresntado em 07/12/2017

Aprovado em 30/09/2018

Versão final apresentada em 02/10/2018 